\title{
292. Fehlerhafte Technik und Komplikationen bei Anwendung des Verriegelungsnagels
}

\author{
M. Börner, K. Klemm, J. Mockwitz und V. Vécsei \\ Berufsgenossenschaftliche Unfallklinik, Friedberger Landstraße 430, D-6000 Frankfurt/M. 60
}

\section{Faulty Technique and Complications in Interlocking Nail Implantation}

Summary. Since 1971, more than 1,000 fractures of the upper and lower leg have been treated with an interlocking nail in the Berufsgenossenschaftliche Unfallklinik Frankfurt/M. Like in every other osteosynthesis system, a faulty technique can be followed by failure and, therefore, this technique can be viewed negatively. Examples of false indications and faulty operating techniques are demonstrated. To avoid these failures, one must know, understand, and master this osteosynthesis technique.

Key words: Interlocking nail - Faulty technique - Complications.

Zusammenfassung. In der Berufsgenossenschaftlichen Unfallklinik Frankfurt/M. haben wir seit 1971 über 1000 Ober- und Unterschenkelfrakturen mit einem Verriegelungsnagel versorgt. Wie bei jedem anderen Osteosyntheseverfahren kann eine fehlerhafte Technik zu Mißerfolgen und damit zu einer Fehleinstellung zu diesem Verfahren führen. Fehler in der Indikationsstellung sowie in der operativen Technik werden anhand von Fallbeispielen demonstriert. Diese Fehler zu vermeiden heißt, diese Osteosyntheseverfahren zu kennen, zu verstehen und zu beherrschen.

Schlïsselwörter: Verriegelungsnagelung - Fehlerhafte Technik - Komplikationen.

\section{Frïherkennung von septischen Komplikationen nach operativer Knochenbruchbehandlung durch Untersuchung der Flüissigkeiten in den Saugdrainagen}

\author{
J. Reinmüller, U. Knapp und F. Schauwecker \\ Unfallchirurgische Klinik, Städt. Kliniken, Ludwig-Erhard-Straße, D-6200 Wiesbaden
}

\section{Early Diagnosis of Pyogenic Infection After Operative Treatment of Bone Fractures by Observation of the Fluids in Wound Suction Drainage}

\begin{abstract}
Summary. Prospective studies on leucocyte concentrations and relative amounts of insoluble components in the fluids of wound suction drainage showed the expected exponential decrease during the first 2 days postoperatively in cases of primary wound healing. When wound healing was complicated by pyogenic infection, however, leucocyte concentrations increased, while the amount of insoluble matter behaved as indicated before. We conclude that leucocyte concentrations in the fluids of wound suction drainage may be used to obtain early prognosis of wound healing.
\end{abstract}

Key words: Leucocyte concentration - Wound suction drainage - Wound healing.

Zusammenfassung. Prospektive Untersuchungen der Leukocytenkonzentration und des Anteils der unlöslichen Bestandteile am Gesamtvolumen in den Flüssigkeiten aus Wund-Saugdrainagen zeigten im Falle primärer Wundheilung erwartungsgemäß eine exponentielle Abnahme über den 1. und 2. postoperativen Tag. Sofern pyogene Wundinfekte auftraten, wurde eine Zunahme der Leukocytenkonzentration beobachtet, während der Anteil der unlöslichen Bestandteile wie beschrieben abnahm. Somit kann durch Bestimmung der Leukocytenkonzentration in den Drainage-Flüssigkeiten frühzeitig eine Prognose bezüglich der Wundheilung gestellt werden.

Schliisselwörter: Leukocytenkonzentration - Wund-Saugdrainage - Wundheilung. 